Artigo/Article

\title{
Tratamento da doença de Gaucher: um consenso brasileiro
} Gaucher disease tr eatment: a brazilian consensus

Ana M. Martins ${ }^{1}$

Clarisse L. Lobo ${ }^{2}$

Elisa A. P. Sobreira ${ }^{3}$

Eugenia R. Valadares ${ }^{4}$

Gilda Porta 5

José Semionato Filho ${ }^{6}$

Mara A. D. Piaonovsky ${ }^{7}$

Marcelo S. Kerstenetzky ${ }^{8}$

Maria F. P. Montoril ${ }^{9}$

Paulo C. Aranda10

Ricardo F. Pires ${ }^{11}$

Ronald M. V. Mota ${ }^{12}$

Teresa C. Bortolheiro ${ }^{13}$

Maria T. M. Paula²

\begin{abstract}
A doença de Gaucher (DG) é um erro inato do metabolismo do grupo das doen ças lisossômicas de depósito, sen do a mais freqüente do referido grupo. É de herança autossômica recessiva, portanto com risco de $25 \%$ a cada gestação de casal heterozigoto. A doença é resultante da deficiência da beta-glicosidase ácida ou beta-glicocerebrosidase, que leva ao acúmulo de glicolipídios nos macrófagos principalmente em baço, fígado, medula óssea e pulmão. As manifestações clínicas ou fenotípicas da DG vão depender do grau de deficiência da enzima, existindo três tipos: Tipo I, forma não neuropática, afeta crianças e adultos com hepatoesplenomegalia, anemia, trombocitopenia, leucopenia elesões ósseas; Tipo II, forma neuropática agu da, afeta crianças com 4-5 meses com quadro neu rológico grave, hepatoesplen omegalia e comprometimento pulmonar e o Tipo III, forma neuropática crônica, afeta crianças e adolescentes com quadro neurológico menos grave que o Tipo II eainda pode comprometer fígado, baço e ossos. Um grupo de catorze médicos com experiência no tratamento da DG com reposição enzimática realizaram extensa revisão da literatura, confrontaram com os dad os evolutivos dos pacien tes brasileiros e chegaram a um consenso quanto aos critérios para iniciar o tratamento, a dose da enzima efreqüência dasinfusões, do acompanhamento ambulatorial, laboratorial eradiológico. O Grupo Brasileiro deEstudosem Doença de Gaucher e outras Doenças de Depósito Lisossômico (GBDDL) tem o objetivo de estabelecer diretrizes para o diagnostico, tratamen to eacompanhamento de pacientes com doença de Gaucher no Brasil. Esta iniciativa pioneira visa uniformizar a conduta no país com relação ao tratamento de DG com reposição enzimática, tratamento de alto custo porém de grande eficácia. Rev. bras. hematol. hemoter. 2003;25(2):89-95.
\end{abstract}

Palavras-chave: Doença de Gaucher; doenças de depósito; Brasil.

\footnotetext{
${ }^{1}$ Ambulatório de Doen ças Metabólicas Hereditárias - Universidade Federal de São Paulo.

Instituto Estadual de Hematologia Arthur de Siqueira Cavalcanti - HEMORIO - Rio de Janeiro-RJ.

${ }^{3}$ Hemato Oncologia Infantil - Santa Casa de Misericórdia de São Paulo.

${ }^{4}$ Departamento de Pediatria da Faculdade de Medicina - Universidade Federal de Minas Gerais.

5 Unidade de Hepatologia Pediátrica - Instituto da Criança do Hospital das Clínicas - Faculdade de Medicina da USP.

Centro Geral de Pediatria - Fundação Hospitalar do Estado de Minas Gerais (FHEMIG).

Disciplina de Hemato-Oncologia Infantil do Departamento de Pediatria - Universidade Federal do Paraná.

${ }^{8}$ Hepatologia Infantil do Hospital da Restauração - Pernambuco.

${ }^{9}$ Fundação Centro de Hemoterapia e Hematologia do Pará (HEMOPA).

${ }^{10}$ Serviço de Hematologia do Hospital Evangélico de Londrina - Paraná.

${ }_{11}^{11}$ Departamen to de Genética Clínica - Hospital de Clínicas de Porto Alegre - Universidade Federal do Rio Gran de do Sul.

${ }_{12}^{12}$ Serviço de Ortopedia Pediátrica - Hospital Sao Bento - Belo Horizonte - Minas Gerais.

${ }^{13}$ Serviço de Hematologia e Oncologia do Depto. de Clínica Medica da Fac. de Ciências Médicas da Sta. Casa de São Paulo.

Grupo Brasileiro de Estudos em Doença de Gaucher e outras Doenças de Depósito Lisossômico (GBDDL)
}

Correspondência para: Ana Maria Martins

Avenida Nove de Julho, 2021/ Apto 133 - Bela Vista - 01313-001 - São Paulo-SP - anamartins.dped@epm.br 


\section{Introdução}

Os erros inatos do metabolismo (EIM) resultam da falta de atividade de uma ou mais enzimas específicas ou defeitos no transporte de proteínas, e produzem manifestações em cada órgão, desde a vida fetal à geriátrica. ${ }^{1,2}$

A incidência acumulativa internacional dos EIM é de 1:5000 dos recém-nascidos vivos. ${ }^{3}$

A doença de Gaucher (DG) é um EIM do grupo das doenças lisossômicas de depósito, sendo a mais freqüente do referido grupo. ${ }^{2} \mathrm{~A}$ herança da doença é autossômica recessiva, portanto, existe risco de recorrência de $25 \%$ a cada gestação do casal de heterozigotos, e pode comprometer filhos de ambos os sexos. ${ }^{1}$

A DG é resultante da deficiência da betaglicosidase ácida ou beta-glicocerebrosidase, que leva ao acúmulo de glicolipídios nos macrófagos, principalmente em baço, fígado, medula óssea e pulmão. A DG ainda pode manifestarse no sistema nervoso central por acúmulo de metabólitos de glicoesfingolipídios endógenos no tecido cerebral. ${ }^{4}$

Os macrófagos repletos das inclusões do substrato têm à microscopia a aparência de "papel amassado" e são as chamadas células de Gaucher, que podem ser encontradas em medula óssea, fígado ou baço. ${ }^{5}$

O Grupo Brasileiro de Estudos em Doença de Gaucher e outras Doenças de Depósito Lisossômico (GBDDL) tem o objetivo de estabelecer diretrizes para o diagnóstico, tratamento e acompanhamento de pacientes com doença de Gaucher e outras doenças de depósito no Brasil. 0 grupo surgiu por um interesse em comum de vários especialistas, é formado por profissionais de área da saúde com experiência no diagnóstico e tratamento da doença de Gaucher. Para participar, deve-se entrar em contato com o grupo já estabelecido através do Fórum DDL (Doença de Depósito Lisossômico - www.forum-ddl.com.br). Há reuniões periódicas e disponibilidade de membros do grupo de visitar e dar assessoria aos serviços interessados. O registro brasileiro já existe e é uma extensão do "Gaucher Registry", gerenciado pelo ICGG (International Colaborative Gaucher Group) e está em atividade no país através dos coordenadores Dr. Ricardo Pires e Dra. Elisa Sobreira. As fichas de registro estão disponí-

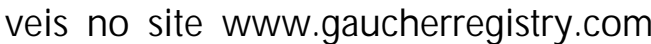

\section{Quadro clínico}

As manifestações clínicas ou fenotípicas da DG vão depender do grau de deficiência da beta-glicosidase ácida e do acúmulo dos glicolipídios, que são variáveis. Existem três fenótipos descritos da DG:

- Tipo 1 (forma não neuropática): Afeta crianças e adultos, a idade de início dos sinais e sintomas é muito variável. A apresentação clínica típica é hepatomegalia, esplenomegalia levando a hiperesplenismo com progressiva anemia, trombocitopenia e leucopenia. 0 quadro é ainda associado à fadiga, cansaço, plenitude gástrica pós-prandial e retardo de crescimento em crianças. 0 acúmulo de glicocerebrosídeo na medula óssea leva à osteopenia, lesões líticas, fraturas patológicas, dor óssea crônica, crises ósseas, infarto e osteonecrose. É descrito também uma maior incidência de tumores ósseos nos pacientes com DG. A progressão do quadro é, em geral, lenta ou variável, e a sobrevida pode ser normal, na dependência da gravidade das complicações. ${ }^{6,7,8}$

0 tipo I é o mais freqüente, correspondendo a $95 \%$ dos casos de DG, tendo uma incidência de 1:10.000 a 1:20.000.9-11

- Tipo 2 (forma neuropática aguda): Afeta lactentes com 4-5 meses de idade, compromete cérebro, baço, fígado e pulmão. 0 quadro neurológico é grave, com múltiplas convulsões, hipertonia, apnéia e progressivo retardo mental. A incidência descrita é menor que 1:100.000. A evolução é rápida, com morte nos primeiros dois anos de vida, em geral pelo envolvimento pulmonar. 6,10

- Tipo 3 (forma neuropática crônica): Afeta crianças e adolescentes, a idade de início é variável, mas em geral no pré-escolar. Compromete cérebro, baço, fígado e ossos. A evolução do quadro neurológico é variável, mas menos grave que o do tipo II. A incidência des- 
crita é menor que 1:100.000. A sobrevida se dá até a segunda ou terceira década de vida. . $^{6,10}$

\section{Diagnóstico}

A suspeita de DG é clínica e o método definitivo de diagnóstico é a dosagem enzimática da atividade da beta-glicosidase ácida. ${ }^{2,7,10}$

Mais de 150 mutações no ácido desoxirribonucleico (DNA), no cromossomo 1 (1q2.1) têm sido descritas, porém existem hoje sete alterações nucleotídeas que respondem pela maioria dos casos. Embora não exista uma perfeita correlação entre o genótipo (tipo de mutação) e o fenótipo, pelo menos é possível distinguir a forma não neuropática da neuropática. A L444P em estado homozigoto (o paciente recebeu a mesma mutação ou alelo do pai e da mãe) está mais associada a sintomas neurológicos precoces, podendo observar-se nos tipos II e III. O alelo N370S está associado com doença não neuropática. ${ }^{6,7,10}$ É importante realizar a pesquisa das mutações nos pacientes, pais e irmãos, para conhecermos melhor a DG no nosso meio, fazer previsões de tratamento e realizar um aconselhamento genético mais seguro. ${ }^{3}$

Critérios de inclusão para o protocolo de tratamento com reposição enzimática ${ }^{10-12}$

1. Diagnóstico confirmado de doença de Gaucher, pela dosagem da atividade enzimática da beta-glicosidadese ácida em leucócitos.

2. Portador de doença de Gaucher do tipo I ou III. Nos pacientes do tipo III, o tratamento deve ser introduzido precocemente, antes de quadro neurológico grave estar estabelecido.

Além dos critérios 1 e 2 descritos acima, deve estar presente pelo menos uma das situações abaixo:

- Apresentar evidências clínicas de comprometimento hepático ou esplênico ou esplenectomia prévia

- Apresentar anemia com hemoglobina abaixo de $2 \mathrm{~g} / \mathrm{dl}$ dos níveis previstos para sexo e idade $/ \mathrm{mm}^{3}$

- Apresentar plaquetas abaixo de 100.000
- Apresentar evidência radiológica de doença óssea (osteopenia, deformidade em frasco Erlenmeyer, osteoartrose, osteoesclerose, osteoporose, fratura patológica, necrose asséptica)

- Apresentar dor óssea ou crises ósseas

- Apresentar retardo de crescimento definido como: crianças com estatura abaixo do percentil 3, abaixo do esperado para o potencial genético da média das alturas dos pais, ou decréscimo na velocidade de crescimento.

- Apresentar sintomas como dor abdominal, fadiga, fraqueza ou caquexia e comprometimento da qualidade de vida

- Comprometimento de outros órgãos (coração, rins e pulmão)

- História de irmão/irmã com doença de Gaucher tipo III com presença de genótipo idêntico

Tratamento com reposição enzimática

- Imiglucerase: cada frasco de $200 \mathrm{UI}$ ou $400 \mathrm{UI}$ deve ser reconstituído em $5,1 \mathrm{ml}$ ou $10,2 \mathrm{ml}$ de água destilada respectivamente e posteriormente diluído em soro fisiológico para um volume final de $200 \mathrm{ml}$

- Via e freqüência: infusão intravenosa a cada 14 dias

- Dose inicial: de acordo com a classificação abaixo:

- Crianças e adultos:

- $30 \mathrm{UI} / \mathrm{kg}$ de peso corpóreo/por infusão

- Crianças e adultos de alto risco:

- $60 \mathrm{UI} / \mathrm{kg}$ de peso corpóreo/por infusão

- Crianças com DG do tipo III fusão

- $120 \mathrm{UI} / \mathrm{kg}$ de peso corpóreo/por in-

- Pacientes de alto risco

- Doença óssea em atividade

- Fígado maior do que 2,5 vezes o valor normal

- Baço maior que 15 vezes o valor normal

- Plaquetas abaixo de $60.000 / \mathrm{mm}^{3}$ ou episódios de sangramento documentados

- Anemia sintomática ou $\mathrm{Hb}$ abaixo ou igual a $8 \mathrm{~g} / \mathrm{dL}$ 
- Doença hepática grave

- Doença renal grave

- Limitação funcional grave caracterizada por doença incapacitante

- Qualquer condição clínica concomitante que possa exacerbar ou complicar a doença de Gaucher.

A dose inicial em adultos e crianças de alto risco deve ser mantida por pelo menos 24 meses, garantindo assim melhora eficaz e prevenção de complicações graves seguindo sempre o juízo clínico do médico que acompanha 0 paciente. ${ }^{24-27}$

0 cuidado deve ser muito mais intenso quando o paciente é criança e já apresenta comprometimento ósseo, mostrando assim a gravidade de seu quadro; este tipo de doente merece especial atenção para prevenção de complicações mais graves na vida adulta, que podem levar até a limitação incapacitante. 7,13,28 Outra peculiaridade da criança é que ela está em fase de crescimento e o comprometimento da estatura final é descrito nos casos não tratados ou tratados com subdoses. ${ }^{14,29}$

- Dose de manutenção:

- Diminui-se a dose nas crianças para até $30 \mathrm{UI} / \mathrm{kg}$ de peso por infusão caso tenha havido normalização dos critérios de inclusão.

- Diminui-se a dose nos adultos para até $20 \mathrm{UI} / \mathrm{kg}$ de peso por infusão caso tenha havido normalização dos critérios de inclusão. dose

- Critério de melhora para diminuição da

- Normalização de todos os critérios utilizados para sua inclusão

- Melhora na qualidade de vida

Acompanhamento clínico e laboratorial ${ }^{7,0}$

As avaliações abaixo sugeridas devem ser realizadas em todos os pacientes com DG em tratamento com reposição enzimática (TRE) ou não:

- Monitoramento dos pacientes com história clínica e exame físico a cada 3/6 meses
- Hemograma completo, fosfatase ácida total, enzimas hepáticas a cada 3/6 meses

- Avaliação volumétrica do fígado e baço preferencialmente por tomografia computadorizada ou ressonância magnética; se não for possível um destes exames, realizar ultra-sonografia abdominal a cada 12 meses

- Avaliação radiológica do esqueleto e/ou densitometria óssea a cada 12 meses ou a critério médico. Pode ser realizada, quando possível, ressonância magnética ponderada $T_{1}$ e $\mathrm{T}_{2}$ do quadril até os joelhos.

0 tratamento dos pacientes portadores de DG, como em outros erros inatos do metabolismo, deve constar de freqüente atualização da literatura por parte dos profissionais, pois um grande número de pesquisas vem sendo realizado no mundo visando à qualidade e eficácia da terapêutica. ${ }^{15}$

São inúmeros os problemas emocionais e sociais que os pacientes portadores de doenças raras enfrentam no seu dia a dia e, por esta razão, devem receber um acompanhamento multiprofissional, consistindo além do clínico, do aconselhamento genético periódico, suportes psicológico, fisioterápico e nutricional. ${ }^{30,31}$

\section{Registro de dados}

Deve existir um registro, ${ }^{13,31}$ como vem sendo realizado em outros países, com o nome do médico responsável, de todos os pacientes portadores de DG, em TRE ou não, porque só desta forma poderemos conhecer a evolução de nossos pacientes com ou sem o tratamento e, a partir destes dados, modificar ou não protocolos de tratamento e acompanhamento de acordo com a experiência brasileira.

\section{Comentários adicionais}

Quando se trabalha com doenças individualmente raras, como são os erros inatos do metabolismo, devemos sempre buscar o conhecimento básico em textos clássicos, que serão o início da trilha que nos levará a um melhor conhecimento da doença. ${ }^{17,18} \mathrm{~A}$ literatura científica é unânime em reconhecer os benefícios 
da TRE nos pacientes portadores de DG, 19,32-36 porém existem controvérsias quanto à dose da enzima recombinante a ser utilizada. $20,22,23,25,37$ ${ }^{40}$ Devido ao custo da enzima, existem inúmeros trabalhos tentando mostrar efeitos similares com baixas doses, porém há falta de rigor nesses relatos, que não separam pacientes graves de leves, adultos de crianças e até colocam no estudo portadores de DG que nem têm indicação de fazer a TRE. ${ }^{19-23,32-40} \mathrm{O}$ uso da TRE com baixas doses em pacientes com DG do tipo tem levado ao aparecimento de graves complicações ósseas nos mesmos. ${ }^{41}$

No futuro, a partir do maior conhecimento dos pacientes de cada região do mundo e dos efeitos da TRE a longo prazo, a dose poderá ser racionalmente individualizada, levando em conta as necessidades do paciente.

0 mais importante é que, ao decidir sobre o tratamento e acompanhamento do paciente com DG, o médico avalie os dados disponíveis na literatura e os resultados da sua experiência, pesando as vantagens e desvantagens para a saúde do paciente, mesmo tendo consciência do alto custo da medicação.

Novas tentativas terapêuticas estão sendo estudadas ${ }^{42}$ e é importante que elas sejam incrementadas, para que no futuro nenhum paciente seja privado de ter uma melhora tão fantástica na qualidade da sua vida, como a que temos observado em nossos pacientes com DG que recebem a TRE, e isto realmente não tem preço.

o Consenso Brasileiro do Tratamento da Doença de Gaucher deverá ser revisto em 2-3 anos, quando teremos maior experiência adquirida com o acompanhamento dos nossos pacientes ou quando surgirem novidades na literatura científica quanto à terapêutica da DG que possa beneficiar nossos pacientes.

Abstract

Gaucher disease is one of the inborn errors of metabolism from lysossomal storage diseases and the most frequent of thisgroup. It issan autosomal recessive disease with reoccurrence risk of $25 \%$ in each pregnancy of hetrozygotes parents. Thedisease results from an b-glucosidase acid or b-glucocerebrosidase deficiency leading to accumulation of glucolipids mainly in the spleen, liver, bone marrow, and lung macrophages. The clinical manifestations of Gaucher disease depend on the enzyme deficiency level. There are three types of the disease, namely, Type I, corresponding to a non-neuropathic form affecting both adults and children with hepatosplenomegaly, anemia, thrombocytopenia, leukopenia, and bone lesions; Typell, corresponding to an acute neuropathic form affecting 4- to 5-month-old babies with a severe neurological condition, hepatosplenomegaly, and lung damage; Type III, corresponding to a chronic neuropathic form affecting both children and teenagers with less severe neurological conditions than Type II besides being able to cause damage to liver, spleen and bones. A group of fourteen physicians experienced in treating Gaucher disease by enzyme replacement therapy has performed an extensive literature review on the subject and compared data of the evolution of Brazilian patients, having reached a consensus as regards the criteria to start treatment, the enzyme doses and frequency of infusions, as well as medical evaluations and laboratory and radiological monitoring. The purpose of this pioneering initiativeis to standardize the conduct within the country, of Gaucher disease treatment using enzymereplacement therapy, which is an expensive treatment but with an expressive efficacy. Rev. bras. hematol. hemoter. 2003;25(2):89-95

Key words: Gaucher disease; deposit disease; Brazil.

\section{Referências Bibliográficas}

1. Martins AM. Inborn Errors of Metabolism: a clinica purview. São Paulo Medical Journal/Rev Paul Med 1999;117(6): 251-65.

2. Scriver CR. Foreword IN: Blau N, Duran M, Blaskovics ME. Physician's Guide to the Laboratory Diagnosis of Metabolic Diseases. 3rd ed. Great Britain 1996.

3. Wilcox WR. Inborn Errors of Metabolism. Online Copyright (C) 1995 World Wide Web URL: http:// www .neonatology.org/syllabus/iem.01.html

4. Cox TM. Gaucher disease: understanding the molecular pathogenesis of sphingolipidoses. J Inherit Metab Dis 2001;24 (Suppl. 2):106-12.

5. MacElwee M, Tanuos $H$, Rogers $S$, Macariola Jr DR Staat, MA, Brady RC. Index of suspicion. Pediatr Rev 2001;22(11):388-93.

6. Barranger JA, O'Rourke E. Lessons learned from the development of enzyme therapy for Gaucher disease. J Inherit Metab Dis 2001;24 (Suppl. 2): 89-96.

7. Charrow J, Esplin JA, Gribble T], Kaplan P, Kolodny EH, Pastores GM et al. Gaucher Disease: recommendations on Diagnosis, Evaluation, and monitoring. Arch Intern Med 1998;158(14):1.754-1.760. 
8. Böhm P, Kunz, W, Horny, H-P, Einsele. Adult Gaucher disease in association with primary malignant bone tumors. Cancer 2001;91:457-462.

9. Altarescu G, Schiffmann R, Parker CC, Moore DF, Kreps $C$, Brady RO et al. Comparative efficacy of dose regimens in enzyme replacement therapy of Type I Gaucher disease. Blood Cells Mol Dis 2000;26(4):285-290.

10. Grabowski GA, Leslie N and Wenstrup R. Enzyme therapy for Gaucher disease: the first 5 years. Blood Rev 1998;12(2):115-133.

11. NIH Technology Assessment: Panel on Gaucher Disease Current issues in diagnosis and treatment. JAMA 1996;275:548-553.

12. Vellodi A, Bembi B, De Villemeur TB, Collin-Husted T, Erikson A, Mengel $E$ et al. Management of neuropathic Gaucher disease: A European consensus. J Inherited Metab Dis 2001:24:319-327.

13. Charrow J, Andersson HC, Kaplan P, Kolodny EH, Mistry $P$, Pastores $G$ et al. The Gaucher Registry: demographics and disease characteristics of 1698 patients with Gaucher disease. Arch Intern Med. 2000;160(9):2.835-2.843

14. Kaplan P, Mazur A, Manor O, Charrow J, Espplin J, Grible TJ et al. Acceleration of retarded growth in children with Gaucher disease after treatment with alglucerase. J Pediatr 1996;129:149-153.

15. Hollak CEM, Maas M, Aerts JM. Clinically relevant therapeutic end points in type I Gaucher disease. J Inherit Metab Dis 2001:24 (Suppl. 2)97-105.

16. Sibille A, Eng CM, Kim SJ, Pastores G, Grabowski GA. Phenotype/genotype correlations in Gaucher disease Type I: clinical and therapeutic implications. Am J Hum Genet 1993:52:1.094-1.101.

17. Beutler E, Grabow ski. Gaucher Disease. IN: Scriver CR, Beaudet AL, Sly WS, Valle D. The Metabolics and Molecular Basis of Inherited Disease, 8th ed., Vol. III. 3.665 -3.668. New York: McGraw-Hill Publishing Company. 2001.

18. Online Mendelian Inheritance in Man, OMIM ${ }^{\mathrm{TM}}$. Center for Medical Genetics, Johns Hopkins University (Baltimore, MD) and National Center for Biotechnology Information, National Library of Medicine (Bethesda, MD), 1997. \#230800 Gaucher Disease, Type I World Wide Web URL:http://www.ncbi.nlm.nih.gov/omim/

19. Grabowski GA, Barton NW, Pastores G, Dambrosia JM, Banerjee TK, McKee MA et al. Enzyme therapy in Type 1 Gaucher disease: Comparative efficacy of mannoseterminated glucocerebrosidase from natural and recombinant sources. Ann Intern Med 1995;122:33-39.

20. Pastores GM, Sibille AR, Grabowski GA. Enzyme Therapy in Gaucher disease Type 1: Dosage efficacy and adverse effects in 33 patients treated for 6 to 24 months. Blood 1993;82:408-416.

21. Moscicki RA, Taunton-Rigby A. Treatment of Gaucher disease - Series of letters. N Engl J Med 1993; 328:1.5641.568 .
22. Hollak AEM, Corssmit EPM, Aerts JMFG, Endert E, Sauerwein $P$, Romijn JA et al. Differential effects of enzyme supplementation therapy on manifestations of Type 1 Gaucher disease. Am J Med 1997;103:185 191

23. Ida $H$, Rennert OM, Kobayashi M, Eto $Y$. Effects of enzyme replacement therapy in thirteen Japanese pediatric patients with Gaucher disease. Eur J Ped 2001; 160:21-25.

24. Rosenthal DI, Doppelt SH, Mankin HJ, Dambrosia JM, Xavier Rj, McKusick KA et al. Enzyme replacement therapy for Gaucher Disease: skeletal responses to macrophage-targeted glucocerebrosidase. Pediatrics 1995;96(4):629-637.

25. Hermann G, Pastores GM, Abdelwahab LF, Lorberboym Am. Gaucher disease: assessment of skeletal involvement and therapeutic responses to enzyme replacement. Skeletal Radiol 1997; 26:687-696.

26. Rodrigue SW, Rosenthal DI, Barton NW, Zarakowiski $D$, Mankin HJ. Risk factors for osteonecrosis in patients with type 1 Gaucher's Disease. Clin Orthop Rel Res 1998;362:201-207.

27. Ida H, Renert OM, Kato S, Ueda T, O ishi K, Maekawa $\mathrm{K}$, Ero $\mathrm{Y}$. Severe skeletal complications in Japanese patients with Type 1 Gaucher disease. J Inherited Metab Dis 1999;22:63-73.

28. Ashkenazi A, Zairov R, Matoth Y. Effect of splenectomy on destructive bone changes in children with chronic (Type I) Gaucher disease. Eur J Pediatr 1986;145:138141.

29. Kauli R, Zaizov R, Lazar L, Pertzelan A, Laron Z, Ma $A G$ et al. Delayed growth and puberty in patients with Gaucher Disease Type 1: natural history and effect of splenectomy and/or enzyme replacement therapy. Is Med Assoc J 2000;2:158-163.

30. Martins AM, D'Almeida V, Tavares FM, Oliveira RB Macedo DM*, Aoki M et al. Características de um ambulatório geral de doenças metabólicas hereditárias. Artigo eletrônico, 1(4) - O nline 1999 - World Wide Web URL: http://www.brazilpednews.org.br

31. Giraldo P, Pocovi M, Perz-Calvo JI, Rubio-Felix D and Giralt M. Report of the Spanish Gaucher's Disease registry: clinical and genetic characteristics. Hematologica 2000;85(8):792-799.

32. Barton NW, Furbish FS, Murray GJ, Garfield M, O'Brady $R$. Therapeutic response to intravenous infusions in patient with Gaucher disease. Proc Natl Acad Sci 1990 87:1.913-1.916.

33. Beutler E, Kay A, Saven A, Garver P, Thurston D, Dawson A, Rosenbloom B. Enzyme replacement for Gauche disease. Blood 1991;78:1.183-1.189.

34. Parker RI, Barton NW, Read EJ, O'Brady R. Hematologic improvement in a patient with Gaucher disease on lon term enzyme replacement therapy: evidence for decreased splenic sequestration and improved red blood cell survival. Amer J Hematol 1991;38:130-137. 
35. Barton NW, Brady, RO, Dambrosia J, Di Bisceglie AM Doppelt, SH hill SC et al. Replacement therapy for inherited enzyme deficiency - macrophage-targeted glucocerebrosidase for Gaucher's disease. N Engl J Med 1991;324:1.464-170.

36. Beutler E. Gaucher disease: New molecular Approaches to diagnosis and treatment. Science. 1992 256:794-799.

37. Figueroa ML, Rosenbloom BE, Kay AC, Garver $P$, Thurston DW, Koziol JA et al. A less costly regimen of alglucerase to treat Gaucher's disease. N Engl I Med 1992;327:1.632-1.636.

38. Beutler E, Demina A, Laubscher K, Garver P, G elbart T, Balicki $D$ et al. The clinically course of treated and untreated Gaucher disease. A study of 45 patients. Blood Cells Mol Dis 1995;21:86-108.

39. Zimran A, Elstein D, Kannai, R Zevin S, Hadas-Halpern, Levy-Lahad, E. Low dose enzyme replacement therapy for Gaucher's disease: effects of age, sex, genotype and clinical features on responses to treatment. Amer j Med 1994;97:3-13.

40. Elstein D, Hadas-Halpern I, Itzchaki M, Lahad A, abrahamov A and Zimran A. Effect of low dose enzyme replacement therapy on bones in Gaucher disease patients with severe skeletal involvement. Blood Cells Mol Dis 1996;22:104-111.

41. Desnick RJ. Personal Communication, Feb 2002.

42. Cox T, Lachman R, Hollak $C$ et al. Novel oral treatment of Gaucher's disease with N-butydeoxynojirimycin (OGT 918) to decrease substrate biosynthesis. Lancet 2000:355(9214):1.481-1.485.

Recebido: 20/07/02

Aceito: 15/12/02 